\title{
Diabetic cystopathy in children and adolescents with Type 1 diabetes mellitus
}

\author{
Mona A. K. Salem ${ }^{1}$, Safinaz A. El Habashy ${ }^{1}$, Dalia N. Toaima ${ }^{1^{*}}$, Hassan S. Shaker ${ }^{2}$, \\ Osama M. A. H. Hetta ${ }^{3}$, Jonair H. A. El Kafy ${ }^{1}$ \\ ${ }^{1}$ Pediatric Department, Ain Shams University, Cairo, Egypt; *Corresponding Author: dtoaima@yahoo.com \\ ${ }^{2}$ Urology Department, Ain Shams University, Cairo, Egypt \\ ${ }^{3}$ Radiodiagnosis Department, Ain Shams University, Cairo, Egypt
}

Received 17 November 2013; revised 15 December 2013; accepted 23 December 2013

Copyright (C) 2014 Mona A. K. Salem et al. This is an open access article distributed under the Creative Commons Attribution License, which permits unrestricted use, distribution, and reproduction in any medium, provided the original work is properly cited. In accordance of the Creative Commons Attribution License all Copyrights (C) 2014 are reserved for SCIRP and the owner of the intellectual property Mona A. K. Salem et al. All Copyright (C) 2014 are guarded by law and by SCIRP as a guardian.

\section{ABSTRACT}

Background: Urinary bladder dysfunction is a major complication of diabetes mellitus and its mechanism has been attributed to autonomic and/or peripheral neuropathy. Objectives: Evaluation of diabetes mellitus and neuropathy effect on the urinary bladder dynamics in children and adolescents with Type 1 diabetes mellitus. Patients and Methods: The study included 80 children and adolescents with Type 1 diabetes for at least 5 years; 60 patients were with manifestations of autonomic and/or peripheral neuropathy and 20 patients were free of either. We assessed both groups for presence of cystopathy by means of uroflowmetry and cystometry. Results: All patients with diabetic neuropathy had abnormal urodynamic test results of variable types and degrees with bladder hypercompliance as the most frequent abnormality. Other urodynamic abnormalities were found in both diabetic patients' groups with no significant difference in frequency. Conclusions: Diabetic neuropathy might be strongly related to urodynamic abnormalities particularly the bladder hypercompliance. Some diabetic patients may have cystopathy in absence of evident neuropathy. This may be due to undetected neuropathy or diabetes induced myopathy of the detrusor muscle.

\section{KEYWORDS}

Diabetic Cystopathy; Type 1 Diabetes; Diabetic Neuropathy; Urinary Bladder; Urodynamic Tests

\section{INTRODUCTION}

Poly-neuropathy is the most common late diabetic complication and is more frequent and severe in Type 1 diabetic population [1]. Diabetic autonomic neuropathy is perhaps occurring as frequent as diabetic peripheral neuropathy, and it is manifested by dysfunction of one or more organ systems (e.g., cardiovascular, gastrointestinal, sudomotor or genitourinary system) [2,3]. Diabetes is associated with an earlier onset and increased severity of urologic complications including urinary bladder dysfunction (diabetic cystopathy) [4]. The mechanism of diabetic cystopathy has been attributed to altered neurological function (autonomic and/or peripheral neuropathy) [5]. Diabetic cystopathy has been classically described as a problem of impaired sensation of bladder fullness, increased bladder capacity, reduced detrusor contractility and impaired bladder emptying with resultant increased postvoiding residual urine [6]. However, the classical characteristics of diabetic cytopathy have not always been observed in diabetic patients who demonstrate varied presentations [7]. Diabetic cystopathy is frequently not recognized by patients and their physicians due to its insidious development and inconspicuous symptoms that may start early in the course of diabetes mellitus, but definite lower urinary tract symptoms do not appear until the disease is in an advanced stage [6,8]. So, the prevention of secondary complications of diabetic cystopathy such as recurrent urinary tract infections, bladder diverticulae, vesicoureteric reflux, nephrolithiasis, and pyelonephritis requires full medical attention as well as early urodynamic diagnosis [9]. Hence, the aim of this work was to evaluate the effects of diabetes and diabetic neuropathy on the urinary bladder of Type 1 diabetic children and adolescents, in whom the urologic complications 
are very likely to occur early.

\section{PATIENTS AND METHODS}

\subsection{Patients}

The present study included 80 children and adolescents suffering from Type 1 diabetes mellitus for 5 years or more and regularly following up in the Specialized Pediatric Diabetes Clinic, Children's Hospital of Ain Shams University, Cairo, Egypt. Sixty diabetic patients with manifestations of autonomic and/or peripheral neuropathy were included as patients' group and twenty diabetic patients free of autonomic or peripheral neuropathy were presented as control patients' group. 300 Type 1 diabetic patients were screened for eligibility criteria and served as the population from which the 80 patients were selected. They included 38 males and 42 females with their ages ranging between $61 / 2$ and 18 years with a mean of 13.58 years. The study was conducted during the period from August, 2006 to April, 2008, inclusive. The study was approved by the ethical committee of Ain Shams University. All patients' parents signed an informed consent agreeing to the nature of the study though many of them didn't approve the urodynamic study. Excluded patients were: patients with history of urologic disease such as stricture urethra, posterior urethral valve and meatal stenosis, patients with neurologic disease such as transverse myelitis or meningomyelocele, patients who underwent previous spinal cord operation or previous pelvic operation that might injure the presacral nerve plexus as surgical correction of imperforate anus, patients with urinary tract infection at time of the study until treated and patients with psychological disorders.

\subsection{Methods}

All patients in the study were subjected to full medical history taking and thorough clinical examination. Full neurologic examination, cardiovascular autonomic function tests and nerve conduction studies were done to select patients with neuropathy to be the patient cases and those without neuropathy to represent the control patients' group. Pelviabdominal ultrasonography, uroflowmetry, urine analysis and urine culture were done to all patients. Among the 80 patients who underwent the study, 36 patients (33 males and 3 females) had completed the urodynamic studies (filling cystometry and pressure-flow study). The remaining 44 patients (5 males and 39 females) had their bladder ultrasonography and uroflowmetry studies normal and the parents refused to subject their children to complete the invasive urodynamic studies.

Cardiovascular autonomic function tests (heart rate response to deep breathing, Valsalva maneuver and standing up as well as blood pressure response to standing up and sustained hand grip) were done in the morning with stable blood glucose level where the fasting blood glucose level was not to exceed $130 \mathrm{mg} / \mathrm{dl}$. Patients were not allowed to exercise or take drugs, alcohol, caffeine and tobacco products for 24 hours before the tests. Patients performed the tests after an overnight fast without taking the morning insulin dose and after doing the tests patients were allowed to take their insulin dose and have their breakfast [10]. The tests were based on measuring the reflex changes in heart rate and blood pressure in response to standardized stimuli [11-14]. The heart rate was measured continuously during the first three procedures by an ordinary ECG monitor. Assessment of autonomic nerve damage was based on the combined results from the previous five tests $[11,15]$. Motor nerve conduction studies for both median and tibial nerves bilaterally were done by the Nicolet Viking Quest 4 Channel apparatus (Viasys Healthcare Neurocare Group USA). Laboratory investigations included: glycated hemoglobin level by quantitative calorimetric determination of glycohemoglobin in whole blood, urine analysis and urine culture.

Pelviabdominal ultrasonography was done with particular emphasis on: urinary bladder wall thickness, estimated bladder weight and postvoid residual urine volume. The sonographer was unaware of patient status in respect to neuropathy.

In order to perform uroflowmetry, patients were asked to drink approximately 1 liter of fluid before and another liter on arrival then they were asked to hold their water until comfortably full. Then, they were allowed to pass their urine into the uroflowmeter in privacy. A graphic recording of the uroflow curve was then obtained. Postvoid residual urine volume was routinely measured after voiding by transurethral catheter.

For performing cystometry, patients were asked to lie supine on a couch in order to pass catheters into the bladder and the rectum to measure the intravesical and the intra-abdominal pressures respectively. During the bladder filling phase, bladder sensation, detrusor activity, bladder compliance, urethral function and bladder capacity were assessed. During the voiding phase, the intravesical pressure and flow rate were measured continuously and were recorded on a graph. It was essential that the clinician related the findings to the symptomatic complaints of the patient. In this way the relevance of the results could be assessed [16]. Both uroflowmetry and cystometry were performed using the Dantec Duet apparatus (Medtronic, USA).

\section{STATISTICAL ANALYSIS}

Data were collected, coded, tabulated then analyzed using SPSS ${ }^{\circledR}$ v12.0 computer software. Numerical va- 
riables were presented as mean and standard deviation (SD) while categorical variables were presented as number of cases and percent. Comparisons of numerical variables were performed with unpaired student " $\mathrm{t}$ " test or one way ANOVA, whenever appropriate. Comparisons of categorical variables were performed by Fisher's exact test or Chi-square test, whenever appropriate. Correlations between variables were estimated by Pearson correlation coefficient. Any difference with $\mathrm{p}$ value $<0.05$ was considered statistically significant.

\section{RESULTS}

In our study, comparing patients' demography and diabetes duration, the neuropathy patients had significantly higher age and duration of diabetes than the non-neuropathy patients. However, there was no significant difference regarding the sex distribution (Table 1).

It was remarkable that only two thirds of patients with diabetic neuropathy (autonomic and/or somatic neuropathy) complained of neuropathy symptoms; while the remaining one third did not complain of any symptoms suggestive of diabetic neuropathy. The experience of lower urinary tract symptoms (both the obstructive and overactive symptoms) was significantly more frequent in the neuropathy than the non-neuropathy diabetic patients. One fifth of the diabetic neuropathy patients suffered from the obstructive urinary symptoms while no one of the non-neuropathy group suffered from these symptoms (Table 2).

The mean value of bladder wall thickness was nearly the same in both neuropathy and non-neuropathy diabetic patients. Similarly, the mean increase in estimated bladder weight in patients with neuropathy and those without was close. However, the mean value of residual urine volume was significantly higher in patients with neuropathy than in patients without as shown in Table 2.

Although only $9.5 \%$ of patients with diabetic neuropathy and $18.2 \%$ of the non-neuropathy groups had diminished maximum flow rate in their uroflowmetry study, all of them were found to have abnormal findings in their urodynamic tests (both invasive and non-invasive tests) and no one had completely normal urodynamic test results. Hypercompliant bladder was the most common finding in those diabetic neuropathy patients, whereas hypocompliance of the bladder was the least common finding as shown in Table 3.

Regarding the uroflowmetry results, the mean values of maximum flow rate, average flow rate and voiding time were slightly higher in patients with neuropathy than in patients without. Meanwhile, the mean value of voiding volume was significantly higher in patients with neuropathy than in patients without neuropathy. Pressure flow studies disclosed that the mean value of maximum cystometric capacity was higher in patients with neuropathy than in patients without neuropathy; but this wasn't statistically significant. The mean value of bladder compliance was significantly higher in patients with neuropathy than in patients without neuropathy (Table 3). There was no significant difference between the neuropathy and the non-neuropathy groups regarding the bladder sensation, overactivity, contractility or outlet obstruction. However, two diabetic patients with neuropathy (8\%) had complete absence of bladder sensation; while no patient without neuropathy had absence of bladder sensation. Similarly, acontractile detrusor was found in one diabetic patient with neuropathy (4.7\%) but not found in any non-neuropathy patient.

The duration of diabetes was found to be significantly related to the ultrasound (U/S) estimated bladder wall thickness and residual urine volume ( $<<0.05$ ). On the other hand, it was not significantly related to the U/S estimated increase in bladder weight. Moreover, the diabetes duration was not significantly related to any uroflowmetry or urodynamic parameter as shown in Table 4.

HbA1c mean values were not significantly different in patients suffering from autonomic neuropathy and delayed NCV as compared to those who did not, and was not found to be significantly related to the U/S findings, uroflow findings or cystometry findings.

\section{DISCUSSION}

In the present study, the mean values of age and disease duration were significantly higher in patients with neuropathy than in patients without neuropathy. The fact that diabetic microvascular complications, including neuropathy, are usually long term complications requiring longer disease duration can explain that [17-21]. There was no gender difference between the two studied groups as sex is not a recognized predisposing factor for development of diabetic neuropathy [18]. This is in

Tab le 1. Comparison of demographic data and diabetes duration between patients with neuropathy and patients without neuropathy.

\begin{tabular}{|c|c|c|c|}
\hline & Control patients (20) & Neuropathy Patients (60) & $\mathbf{P}$ \\
\hline Age in yrs (mean \pm SD) & $11.61 \pm 3.27$ & $14.18 \pm 2.96$ & $0.002^{*}$ \\
\hline Diabetes Duration in yrs (mean $\pm \mathrm{SD}$ ) & $6.37 \pm 1.29$ & $8.51 \pm 2.58$ & $<0.001^{*}$ \\
\hline \multirow[b]{2}{*}{ Female } & $11(55 \%)$ & $27(45 \%)$ & \multirow{2}{*}{0.453} \\
\hline & $9(45 \%)$ & 33 (55\%) & \\
\hline
\end{tabular}


Tab le 2. Comparison of clinical data, U/S and uroflowmetric findings between diabetic patients with and without neuropathy.

\begin{tabular}{cccc}
\hline & Non-neuropathy patients (n = 20) & Neuropathy patients (n = 60) & P \\
\hline & Symptoms & & \\
Lower urinary tract Symptoms & $2(10 \%)$ & $22(36.7 \%)$ & $0.026^{*}$ \\
Obstructive Symptoms & $0(0 \%)$ & $12(20 \%)$ & $0.031^{*}$ \\
Overactive Symptoms & $2(10 \%)$ & $15(25 \%)$ & 0.214 \\
& Ultrasonographic findings & & \\
Bladder wall thickness (mm) & $3.10 \pm 1.39$ & $3.09 \pm 1.11$ & 0.970 \\
Increase in estimated bladder weight (gm) & $20.70 \pm 16.40$ & $24.4 \pm 22.01$ & 0.493 \\
Residual volume (cc) & $13.73 \pm 14.66$ & $33.71 \pm 42.02$ & $0.041^{*}$ \\
Bladder wall thickness (mm) & Uroflowmetric findings & & 0.970 \\
Voiding volume (ml) & $3.10 \pm 1.39$ & $3.09 \pm 1.11$ & $0.031^{*}$ \\
Maximum cystometric capacity (cc) & $365.61 \pm 183.70$ & $496.96 \pm 302.17$ & 0.652 \\
Compliance & $468.36 \pm 186.20$ & $496.6 \pm 164.74$ & $113.44 \pm 89.61$ \\
\hline
\end{tabular}

Tab le 3. Frequency of urodynamic findings among 2 patients' groups.

\begin{tabular}{|c|c|c|c|c|c|}
\hline Urodynamic findings & & Patients with diabetic neuropathy & Patients without neuropathy & chi square & $\mathbf{P}$ \\
\hline \multirow{2}{*}{ Diminished max flow rate } & Yes & $2(9.5 \%)$ & $2(18.2 \%)$ & \multirow{2}{*}{0.49} & \multirow{2}{*}{0.48} \\
\hline & No & 19 & 9 & & \\
\hline \multirow{2}{*}{$\begin{array}{l}\text { Diminished or absent } \\
\text { bladder sensation }\end{array}$} & Yes & $11(44 \%)$ & $5(45.5 \%)$ & \multirow{2}{*}{0.01} & \multirow{2}{*}{0.93} \\
\hline & No & 14 & 6 & & \\
\hline \multirow{2}{*}{$\begin{array}{l}\text { Abnormally high max } \\
\text { cystometric capacity }\end{array}$} & Yes & $17(68 \%)$ & $5(45.5 \%)$ & \multirow{2}{*}{1.63} & \multirow{2}{*}{0.21} \\
\hline & No & 8 & 6 & & \\
\hline \multirow{2}{*}{ Detrusor overactivity } & Present & $6(24 \%)$ & $3(27.3 \%)$ & \multirow{2}{*}{0.04} & \multirow{2}{*}{0.83} \\
\hline & Absent & 19 & 8 & & \\
\hline \multirow{2}{*}{$\begin{array}{l}\text { Hypocontractile and } \\
\text { acontractile detrusor }\end{array}$} & Present & $4(19 \%)$ & $3(42.9 \%)$ & \multirow{2}{*}{0.16} & \multirow{2}{*}{0.67} \\
\hline & Absent & 17 & 4 & & \\
\hline \multirow{2}{*}{ Hypocompliant bladder } & Present & $1(4 \%)$ & $0(0 \%)$ & \multirow{2}{*}{0.45} & \multirow{2}{*}{0.5} \\
\hline & Absent & 24 & 11 & & \\
\hline \multirow{2}{*}{ Hypercompliant bladder } & Yes & $23(92 \%)$ & $10(90.9 \%)$ & \multirow{2}{*}{0.01} & \multirow{2}{*}{0.91} \\
\hline & No & 2 & 1 & & \\
\hline \multirow{2}{*}{ Bladder outlet obstruction } & Yes & $6(33.3 \%)$ & 3 (42.9\%) & \multirow{2}{*}{0.2} & \multirow{2}{*}{0.66} \\
\hline & No & 12 & 4 & & \\
\hline
\end{tabular}

Tab le 4. Relation of diabetes duration to ultrasonographic and urodynamic findings in diabetic patients.

\begin{tabular}{ccc}
\hline & Findings & Diabetes duration \\
\hline Bladder wall thickness & Correlation Coefficient & 0.263 \\
& P & $0.018^{*}$ \\
Increase in bladder weight & Correlation Coefficient & 0.176 \\
& $\mathrm{P}$ & 0.119 \\
Residual volume & Correlation Coefficient & 0.272 \\
& $\mathrm{P}$ & $0.015^{*}$ \\
Max flow rate & Correlation Coefficient & -0.079 \\
& $\mathrm{P}$ & 0.502 \\
Average flow rate & Correlation Coefficient & -0.052 \\
& $\mathrm{P}$ & 0.661 \\
Voided volume & Correlation Coefficient & 0.010 \\
& $\mathrm{P}$ & 0.935 \\
Voiding time & Correlation Coefficient & -0.056 \\
Max cystometric capacity & $\mathrm{P}$ & 0.637 \\
Compliance & Correlation Coefficient & 0.006 \\
\hline
\end{tabular}


agreement with Karavanaki et al. [22] who studied 129 children with Type 1 diabetes in a longitudinal study for the evolution of microvascular disease and detected no pubertal or sex effect on the development of diabetic autonomic neuropathy.

On analyzing patients' symptoms, it was remarkable to find that one third of diabetic patients suffering from neuropathy had no symptoms suggestive of somatic or autonomic neuropathy at all. This is in accord with other studies which proved that patients with diabetic neuropathy were rarely symptomatic but their physical examination revealed mild to moderately severe sensory loss $[21,23]$. Lower urinary tract symptoms, particularly the obstructive symptoms, occurred more frequently in patients with diabetic neuropathy than diabetic patients without neuropathy. This was statistically significant and agreed with Blaivas [24] who documented that the obstructive urinary symptoms (straining, intermittency, postvoid dribbling and weak stream) occur in diabetic patients due to denervation of the bladder and poor detrusor contractility.

Ultrasonography provided a simple non-invasive method for assessment of urologic complications in our studied patients. The mean value of ultrasound estimated residual urine volume was significantly higher in patients with diabetic neuropathy than those without. This is consistent with other studies where the presence of residual urine was strongly associated with peripheral neuropathy $[25,26]$. However, the mean values of ultrasound estimated bladder wall thickness and increase in bladder weight in patients with diabetic neuropathy were close to those of patients without neuropathy. This is in agreement with Szabo et al. [27]. The increase in bladder weight and thickness in both patients' groups can be attributed to diabetes itself as a compensatory mechanism to hyperglycemia and polyuria.

Uroflowmetry was abnormal in small number of patients in each group; and thus it could not detect the abnormal bladder function that was actually present in all of them. This finding is in accord with Szabo and Fegyverneki [28].

Analysis of the results of urodynamic testing revealed that all studied patients with diabetic neuropathy had abnormal urodynamic test results of variable types; though only $36.7 \%$ of them reported lower urinary tract symptoms. This finding may indicate that diabetic neuropathy is strongly related to the presence of diabetic cystopathy; which can occur silently in the course of diabetes and symptoms do not appear until the disease is in advanced stage. The bladder hypercompliance was the most frequent abnormal urodynamic finding present in diabetic neuropathy patients. In addition, the mean value of bladder compliance was significantly higher in patients with neuropathy than in patients without neuropa- thy. This is consistent with a previous study which found that increased bladder compliance was an important factor in diabetic cystopathy [29]. The mean value of voided volume was significantly higher in patients with neuropathy than in patients without. This is in agreement with Barkai and Szabo [17] who found that voided volumes were higher in diabetic patients with cardiac autonomic dysfunction than in those without. The impaired diabetic bladder sensation, that allows accumulation of abnormally large volume of urine before the desire is felt, may be the cause. The other urodynamic findings, which are diminished bladder sensation, weak detrusor contractility, detrusor overactivity and bladder outlet obstruction, were found in both diabetic patients' groups with no significant difference. Yet, the complete absence of bladder sensation and contractility was found only in diabetic patients with neuropathy. The latter finding may indicate that the presence of diabetic neuropathy is associated with the severity of bladder dysfunction. The presence of diminished bladder sensation in patients of the nonneuropathy group may suggest the presence of subclinical autonomic neuropathy that could not be detected. A similar finding was discovered by Barkai and Szabo [17].

The detrusor overactivity and the weak bladder contractility found in patients without neuropathy may be also explained by the presence of subclinical autonomic dysfunction or the presence of myogenic factors rather than neuropathic factors i.e. there is initial adaptation of the diabetic bladder to polyuria by compensatory increase in detrusor activity independent to the presence of neuropathy [7]. Then after a while, chronic overstretching of the bladder may cause myogenic detrusor hypocontractility [9]. This is in agreement with Ueda et al. [26] who found that diabetic patients had weaker bladder contractility compared to healthy controls and this weakness was more pronounced in those without autonomic neuropathy. As regards the bladder outlet obstruction found in non-neuropathy patients, it can also be explained by the presence of subclinical neuropathy affecting the innervation of the external urethral sphincter and leading to detrusor sphincter dyssynergia.

Studying the relationship between the diabetes duration and diabetic cystopathy, it was found that the duration of diabetes was significantly related to the increased U/S estimated bladder wall thickness and residual urine volume ( $<0.05)$; but it was not related to any urodynamic finding indicating that disturbed bladder function may be independent of the duration of diabetes. This is in concordance with the formerly mentioned study of Karavanaki et al. [22] which had findings suggesting that autonomic function can be impaired independent of diabetes duration.

HbA1c is a good indicator of the state of glycemic control. Yet, our patients' mean values of HbA1c were 
not related to the presence of neuropathy, ultrasound or urodynamic abnormalities. This might be attributed to the fact that HbA1c was done once during the course of the study as most of the patients were non-compliant and had no regular record of their HbA1c values.

\section{CONCLUSION}

It is important to note that our study encountered many obstacles that limited the recruitment of larger numbers of patients. These obstacles included financial limitations due to the high cost of urodynamic testing and ethical limitations due to refusal of many patients to complete the urodynamic tests. The lack of background of the early effects of diabetes on the urinary bladder and the fear of invasiveness of the urethral catheter discouraged many patients to join the study. In conclusion, diabetic neuropathy is strongly related to the presence of urodynamic abnormalities of any kind, particularly the bladder hypercompliance. Some diabetic patients may have urodynamic abnormalities in absence of evident neuropathy. This may be due to subclinical autonomic neuropathy, occurrence of dysfunction of the bladder innervations independent of cardiovascular autonomic dysfunction or direct myogenic effect of hyperglycemia and polyuria on the detrusor muscle.

\section{ACKNOWLEDGEMENTS}

The study was supported in part by the Egyptian Ministry of Health. Thanks are due to Professor Sahar A. Hassanein, Professor of Pediatrics, Faculty of Medicine, Ain Shams University, Cairo, Egypt for kindly performing the nerve conduction studies of the patients; Dr. Ahmed Saafan, Lecturer of Urology and Dr. Wael Fawzy, Assistant Lecturer of Urology, Faculty of Medicine, Ain Shams University, Cairo, Egypt for performing the urodynamic studies for the patients.

\section{REFERENCES}

[1] Sima, A.A. (2006) Pathological mechanisms involved in diabetic neuropathy: Can we slow the process? Current opinion in investigational drugs, 7, 324-337.

[2] Kamalakannan, D., Baskar, V. and Singh, B.M. (2004) Severe and disabling diabetic autonomic neuropathy. Journal of Diabetes and its Complications, 18, 126-128. http://dx.doi.org/10.1016/S1056-8727(03)00004-7

[3] American Diabetes Association and American Academy of Neurology. (1988) Report and recommendations of the San Antonio Conference on diabetic neuropathy (Consensus Statement). Diabetes, 37, 1000-1004. http://dx.doi.org/10.2337/diab.37.7.1000

[4] Brown, J.S., Wessells, H., Chancellor, M.B. et al. (2005) Urologic Complications of Diabetes. Diabetes Care, 28, 177-185. http://dx.doi.org/10.2337/diacare.28.1.177

[5] Poladia, D.P., Schanbacher, B., Wallace, L.J. and Bauer, J.A. (2005) Innervation and connexin isoform expression during diabetes-related bladder dysfunction: Early structural vs. neuronal remodeling. Acta Diabetologica, 42, 147-152. http://dx.doi.org/10.1007/s00592-005-0194-y

[6] Sasaki, K., Chancellor, M.B., Phelan, M.W., Yokoyama, T., Fraser, M.O. and Seki, S. (2002) Diabetic cystopathy correlates with a long-term decrease in nerve growth factor levels in the bladder and lumbosacral dorsal root ganglia. Journal of Urology, 168, 1259-1264. http://dx.doi.org/10.1016/S0022-5347(05)64636-8

[7] Daneshgari, F., Liu, G. and Imrey, P.I. (2006) Time Dependent Changes in Diabetic Cystopathy in Rats include Compensated and decompensated Bladder Function. The Journal of Urology, 176, 380-386. http://dx.doi.org/10.1016/S0022-5347(06)00582-9

[8] Lee, W.C., Wu, H.P., Tai, T.Y., Liu, S.P., Chen, J. and Yu, H.J. (2004) Effects of diabetes on female voiding behavior. The Journal of Urology, 172, 989-992. http://dx.doi.org/10.1016/S0022-5347(06)00582-9

[9] Hampel, C., Gillitzer, R., Pahernik, S., Melchior, S. and Thuroff, J.W. (2003) Diabetes mellitus and bladder function. What should be considered? Der Urologe, 42, 15561563. http://dx.doi.org/10.1007/s00120-003-0456-7

[10] Levitt, N.S., Stansberry, K.B., Wynchank, S. and Vinik, A.I. (1996) The natural progression of autonomic neuropathy and autonomic function tests in a cohort of people with IDDM. Diabetes Care, 19, 751-754. http://dx.doi.org/10.2337/diacare.19.7.751

[11] Ewing, D.J. (1992) Analysis of heart rate variability and other non-invasive tests with special reference to diabetes mellitus. In: Bannister, R. and Mathias, C.J. Eds., Autonomic Failure, 3rd Edition. Oxford Medical, Oxford, 312333.

[12] Barkai, L. and Madacsy, A. (1995) Cardiovascular autonomic dysfunction in diabetes mellitus. Archives of Disease in Childhood, 73, 515-518. http://dx.doi.org/10.1136/adc.73.6.515

[13] Cechetto, D.F. (2000) Autonomic nervous system assessment. In: Terhost, G.J. and Zipes, D.P. Eds. The Nervous System and the Heart, 1st Edition. Humana Press, Totowa, 172-177.

[14] Scott, W.A. (2001) Assessment of the autonomic nervous system. In: Allen, H.D., Gutgesell, H.P., Clark, E.B. and Driscoll, D.J. Eds. Moss and Adams' Heart Disease in Infants, Children and Adolescents Including the Fetus and Young Adult, 6th Edition. Lippincott Williams \& Wilkins, Baltimore, 172-176.

[15] Neumann, C. and Schmidt H. (1995) Relationship between the degree of cardiovascular autonomic dysfunction and symptoms of neuropathy and other complications of diabetes mellitus. Brazilian Journal of Medical and Biological Research, 28, 751-757.

[16] Nitti, V.W. (2005) Pressure flow urodynamic studies: The gold standard for diagnosing bladder outlet obstruction. Reviews in Urology, 7, S14-S21.

[17] Barkai, L. and Szabó, L. (1993) Urinary bladder dysfunction in diabetic children with and without subclinical cardiovascular autonomic neuropathy. European Journal of Pediatrics, 152, 190-192. 
http://dx.doi.org/10.1007/BF01956141

[18] Boulton, A.J., Vinik, A.I, Arezzo, J.C. et al. (2005) Diabetic neuropathies: A statement by the American Diabetes Association. Diabetes Care, 28, 956-962.

http://dx.doi.org/10.2337/diacare.28.4.956

[19] Donaghue, K.C., Chiarelli, F., Trotta, D., Allgrove, J. and Dahl-Jorgensen, K. (2007) ISPAD clinical practice consensus Guidelines (2006-2007): Microvascular and macrovascular complications. Pediatric Diabetes, 8, 163-170. http://dx.doi.org/10.1111/j.1399-5448.2007.00250.x

[20] Karavanaki, K. and Baum, J.D. (2003) Coexistence of impaired indices of autonomic neuropathy and diabetic nephropathy in a cohort of children with Type 1 diabetes mellitus. Journal of Pediatric Endocrinology and Metabolism, 16, 79-90.

http://dx.doi.org/10.1515/JPEM.2003.16.1.79

[21] Nokleyby, K. and Berg, T.J. (2005) Diabetic neuropathy-A clinical review. Tidsskr Nor Laegforen, 125, 16461649.

[22] Karavanaki, K., Davies, A.G., Morgan, M.H. and Baum, J.D. (1997) Autonomic function in a cohort of children with diabetes. Journal of Pediatric Endocrinology and Metabolism, 10, 599-607. http://dx.doi.org/10.1515/JPEM.1997.10.6.599

[23] Novella, S.P., Inzucchi, S.E. and Goldstein, J.M. (2001) The frequency of undiagnosed diabetes and impaired glucose tolerance in patients with idiopathic sensory neuropathy. Muscle Nerve, 24, 1229-1231.

http://dx.doi.org/10.1002/mus.1137

[24] Blaivas, J.G. (2002) Cause and effect of lower urinary tract symptoms. Journal of Urology, 168, 1464. http://dx.doi.org/10.1016/S0022-5347(05)64474-6

[25] Beylot, M., Marion, D. and Noel, G. (1982) Ultrasonographic determination of residual urine in diabetic subjects: relationship to neuropathy and urinary tract infection. Diabetes Care, 5, 501-505. http://dx.doi.org/10.2337/diacare.5.5.501

[26] Ueda, T., Yoshimura, N. and Yoshida, O. (1997) Diabetic cystopathy: Relationship to autonomic neuropathy detected by sympathetic skin response. Journal of Urology, 157, 580. http://dx.doi.org/10.1016/S0022-5347(01)65209-1

[27] Szabo, L., Barkai, L. and Lombay, B. (2007) Urinary flow disturbance as an early sign of autonomic neuropathy in diabetic children and adolescents. Neurourology and Urodynamics, 26, 218-221. http://dx.doi.org/10.1002/nau.20349

[28] Szabo, L. and Fegyverneki, S. (1995) Maximum and average urine flow rates in normal children-The miskolc nomograms. British Journal of Urology, 76, 16-20.

[29] Gong, Y., Song, B., Jin, X. and Xiong, E. (2000) Changes in bladder compliance and detrusor contraction/relaxation of diabetic rats. Zhonghua Wai Ke Za Zhi, 38, 865-867. 